\title{
ANÁLISIS DE LA ACUICULTURA DE SALMÓNIDOS INTENSIVA DE GRAN ESCALA EN EL CANAL BEAGLE COMO ESTRATEGIA PARA EL DESARROLLO DE TIERRA DEL FUEGO ${ }^{\circ} \wedge$
}

\author{
ANALYSIS OF LARGE-SCALE INTENSIVE SALMONID \\ AQUACULTURE IN THE BEAGLE CHANNEL AS A STRATEGY \\ FOR THE DEVELOPMENT OF TIERRA DEL FUEGO
}

\author{
Juan Ignacio García* \\ Carolina Hernández* \\ Silvina A. Romano*
}

enviado: 01 noviembre 2019 - aceptado: 07 abril 2020

\section{Resumen}

La posibilidad de radicar proyectos de acuicultura con salmones del Atlántico en las costas argentinas del canal Beagle pone de manifiesto tensiones entre diferentes estrategias de desarrollo. Por un lado, aquella que visualiza la oportunidad de aprovechar el potencial de un recurso natural para generar empleo e ingresos. Por otro lado, aquella que considera que los potenciales beneficios pueden no ser tales si en el análisis se toman en cuenta cuestiones de tipo ambiental, la degradación de los espacios naturales y los potenciales efectos negativos sobre otras actividades productivas. El presente trabajo pone en discusión cuestiones relativas a la efectiva apropiación de los potenciales beneficios de esta actividad desde una perspectiva territorial.

Código JEL: Q2, L7, O1.

- García, J. I., Hernández, C., y Romano, S. A. (2020). Análisis de la acuicultura de salmónidos intensiva de gran escala en el Canal Beagle como estrategia para el desarrollo de Tierra del Fuego. Estudios económicos, 37 (74), 161-190.

^ Este trabajo toma como punto de partida el análisis económico realizado por Juan Ignacio García y Carolina Hernández en 2019: “Bases para un análisis económico de la acuicultura de salmónidos en la costa argentina del canal Beagle”, Universidad Nacional de Tierra del Fuego. Realizado en el marco de discusiones con organizaciones sociales iniciadas en 2018.

* Instituto de Desarrollo Económico e Innovación, Universidad Nacional de Tierra del Fuego. Correo electrónico: jigarcia@untdf.edu.ar, chernandez@untdf.edu.ar, sromano@untdf.edu.ar 
Palabras Clave: acuicultura, salmónidos, desarrollo, tensiones.

\begin{abstract}
The possibility of filing Atlantic salmon aquaculture projects on the Argentine coasts of the Beagle Channel highlights tensions between different development strategies. On the one hand, the one that visualizes the opportunity to take advantage of the potential of a natural resource to generate employment and income. On the other hand, the one that considers that the potential benefits may not be such if the analysis considers environmental issues, the degradation of natural spaces and the potential negative effects on other productive activities. The present work discusses issues related to the effective appropriation of the potential benefits of this activity from a territorial perspective and a specific socio-temporal context.
\end{abstract}

JEL Code: Q2, L7, O1.

Keywords: aquaculture, salmonids, develop, tensions. 


\section{INTRODUCCIÓN}

La posibilidad de radicar proyectos de acuicultura con salmones del Atlántico en las costas argentinas del canal Beagle pone de manifiesto tensiones entre diferentes estrategias de desarrollo. Por un lado, aquella que visualiza la oportunidad de aprovechar el potencial de un recurso natural para generar empleo e ingresos a través de una actividad inserta en una cadena global de valor (CGV). Por otro lado, la que considera que los potenciales beneficios pueden no ser tales si en el análisis se toman en cuenta aspectos de tipo ambiental, la degradación de los espacios naturales y los potenciales efectos negativos sobre otras actividades productivas.

La discusión conceptual en torno a este problema de investigación versa no solo alrededor de la idea de desarrollo sustentable (Informe Brundtland, 1987), sino también respecto al concepto de extractivismo, o neoextractivismo (Gudynas, 2014; Escobar, 2011). Estos marcos conceptuales invitan a revisar los análisis económicos tradicionales desde una postura crítica, incorporando explícitamente la relación entre el medio ambiente y el desarrollo productivo, así como el reconocimiento explícito de los espacios naturales compartidos (Cantero y Romano, 2018) y los conflictos sociales que pueden suscitarse alrededor del uso de estos (Svampa, 2012).

El principal objetivo de este trabajo es aportar a la discusión en torno al potencial económico y los riesgos asociados a la posible radicación de proyectos de acuicultura de salmónidos en la costa argentina del canal Beagle a la luz de una estrategia de desarrollo provincial.

Si bien existen sistemas de producción de salmónidos en tierra u offshore, para este análisis se tomó como referencia el modelo de producción a gran escala, caracterizado por el uso de jaulas para engorde en zonas costeras y fiordos. Esta decisión se justifica no solo por ser este el modo habitual de desarrollo de la actividad, sino también porque durante 2018, a través de la firma de un convenio de cooperación, las entonces autoridades provinciales manifestaban su interés por comenzar estudios orientados a la radicación de inversiones con esta finalidad ${ }^{1}$.

1 En el año 2018 el gobierno de Tierra del Fuego y la Fundación Argentina para la Promoción de Inversiones y Comercio Internacional firmaron un Convenio de Cooperación, con la participación y financiamiento de la agencia “Innovation Norway”, para la realización de un estudio de cargas y posiciones para el desarrollo de la acuicultura de salmones del Atlántico en el Canal Beagle (Convenio N 18270, del 20 de abril de 2018 aprobado por Decreto Provincial 1.332/2018). 
El trabajo se organiza en cuatro secciones. En la primera de ellas se abordará el marco teórico. En la segunda la metodología. En la sección 3 se realizará una descripción de la actividad sobre la base del caso chileno, con acento en la región de Magallanes. En la sección 4 se abordará el caso de la costa argentina del canal Beagle.

\section{MARCO CONCEPTUAL}

La discusión conceptual en torno a este problema de investigación versa alrededor de dos cuestiones que exceden la mirada ortodoxa del crecimiento económico.

Por un lado, el desarrollo sustentable (Informe Brundtland, 1987), entendido como el proceso que puede mejorar la condición de vida de las personas "sin comprometer la capacidad de las generaciones futuras para satisfacer sus propias necesidades”. Existen numerosos trabajos (Martínez, 2007; Romero \& Vázquez, 2003; Guimarães, 1994) que discuten el marco sustentable del desarrollo, en particular en el uso de recursos naturales, involucrando diversas dimensiones como la económica, social, cultural y política. Si bien no existe una definición inequívoca, se entiende que estas múltiples dimensiones requieren mecanismos que regulen su uso.

Por otro lado, la idea de estrategias de desarrollo apalancadas en recursos naturales. Autores como Carlota Pérez (2010) han entendido que los recursos naturales pueden ser una estrategia exitosa de inserción internacional para América Latina, en tanto su desarrollo se logre a través de cadenas de valor internacionales. Sin embargo, desde otra perspectiva, autores como Arturo Escobar (2011) señalan que estas estrategias constituyen una justificación al extractivismo con escaso desarrollo local.

En esta línea, el concepto de extractivismo es utilizado en la literatura para abordar el análisis de proyectos basados en recursos naturales a gran escala. Desde esta perspectiva, se identifica un modelo productivo caracterizado por una fuerte concentración económica de empresas extranjeras intensivas en capital que, a través de la especialización productiva en algún commodity (Svampa, 2012), siguen una lógica de explotación económica basada en la transnacionalización y la subordinación del Estado (Escobar, 2011).

Con el consenso de los commodities, Svampa (2012) se refiere a la situación que se observa en América Latina donde la extracción de recursos naturales se realiza sin agregado de valor por parte de empresas extranjeras, con bajo o escaso desarrollo de encadenamientos locales y riesgo de reprimarización de estas econo- 
mías, las que se ven atravesadas por procesos que evidencian tensiones respecto a la tenencia de la tierra, el uso de bienes comunes (Ostrom et al., 1999) y el desarrollo de otros emprendimientos productivos locales, generando conflictos sociales en torno al uso de estos recursos (Svampa, 2012; Gudynas, 2009; Leff, 2002).

En este trabajo se entiende la necesidad de enriquecer la visión del desarrollo sustentable a la luz de las experiencias latinoamericanas analizadas bajo la perspectiva extractivista. Entendiendo que desde la visión del desarrollo sustentable se establecen normativamente cuestiones que no logran captar la complejidad de la magnitud territorial, consideramos que desde el extractivismo se explicitan tensiones territoriales en torno al uso de los recursos naturales, las que deben ser incluidas en el análisis de una estrategia de desarrollo territorial.

En ese marco, se analizarán las posibilidades reales de apropiación de rentas y generación de capacidades productivas y tecnológicas locales, así como los daños y riesgos ambientales involucrados en el desarrollo de la actividad. Adicionalmente, se analizará el rol que el Estado debería asumir en el plano regulatorio e institucional.

\section{METODOLOGÍA}

A fin de reflexionar sobre la posible instalación de esta actividad en las costas argentinas del canal Beagle, se utilizó como marco de referencia el caso chileno, observando principalmente la experiencia de la región de Magallanes, desde donde se identificaron los rasgos centrales que permitieron caracterizar el posible modelo productivo. El abordaje de estudio de caso se entiende como una estrategia metodológica que admite analizar situaciones que requieren un amplio contenido descriptivo para dar cuenta de las complejidades (Sautú et al., 2005).

En este trabajo se utilizó una combinación de técnicas cualitativas (análisis documental de informes de acceso público y notas periodísticas, entrevistas en profundidad con informantes clave y análisis legislativo), con técnicas cuantitativas (estadística descriptiva de fuentes disponibles).

Para abordar el caso se analizaron cada uno de los eslabones productivos, con especial énfasis en sus interacciones, requerimientos de recursos y la capacidad de generar valor; todo ello desde una mirada global, entendiendo que cada etapa productiva puede estar ubicada en distintos espacios geográficos supra nacionales (Padilla, 2014). 
Asimismo, distintos autores (Borello, 2015; Kaplinzky \& Morris, 2009; Gereffi, 2003; Padilla, 2014) sintetizan elementos relevantes a ser considerados en el análisis de estudios de caso. En este trabajo se hará especial énfasis en el contexto, los encadenamientos productivos (características y requerimientos) y el marco normativo e institucional que organiza la actividad. Adicionalmente, a la luz del marco conceptual utilizado, se hará hincapié en los problemas económicos, ambientales y sociales derivados del desarrollo de la actividad.

\section{DESCRIPCIÓN DE LA ACTIVIDAD SOBRE LA BASE DEL CASO CHI- LENO, CON ACENTO EN LA REGIÓN DE MAGALLANES}

\section{III.1. Contexto}

La mayor parte de la producción mundial de salmones se localiza en pocos países

cadena productiva muestra una tendencia a la concentración económica, donde las empresas noruegas dominan a escala global. Aunque las actividades de I+D se centralizan en dicho país, dominando la producción de alevines y los desarrollos genéticos, la experiencia chilena demuestra que existe lugar para la participación de nuevos actores, en tanto que, con inversiones mucho menores, ha participado incipientemente en la generación de conocimiento requeridos por el sector (Zanlungo et al., 2015).

La exportación de salmónidos, y en particular del salmón Atlántico de piscicultura, constituye el segundo rubro de exportaciones en Chile, después del cobre. El desarrollo de la industria salmonera en la región de Los Lagos data de 1991², momento en el cual se aprueba la Ley General de Pesca y Acuicultura(LGPA), se crean las Áreas Aptas para la Acuicultura (AAA), y comienzan a otorgarse las concesiones acuícolas 3 .

Katz y Araya (2015) señalan la falta de percepción inicial respecto a las diferencias de la pesca industrial y la acuicultura, las que requieren modelos regulatorios y de monitoreo de impacto ambiental distintos. Pasarían diez años hasta modificar la regulación que separa la pesca y la acuicultura, otorgando a la segunda un estatus específico y una serie de instituciones y normas propias de una industria

2 El D. S. N. 430 de 1991 del Ministerio de Economía de Chile fija el texto refundido, coordinado y sistematizado de la Ley 18892 de 1989 y sus modificaciones, como Ley General de Pesca y Acuicultura (Martínez \& Soto, 2016).

3 Según la Fundación Terram, existen autorizaciones anteriores a 1991 y que no se rigen por esta ley. 
que cultiva peces en cautiverio, a diferencia de otra que explota la pesca industrial de altura. En 2001 se dicta el Reglamento Ambiental de la Acuicultura (RAMA) y el Reglamento de Medidas de Protección, Control y Erradicación de Enfermedades de Alto Riesgo para las Especies Hidrobiológicas (RESA).

En estos años, la industria salmonera de Chile creció, transformándose en un importante proveedor internacional de salmones y truchas en sus diversas formas de presentación. Pero este crecimiento se asentó sobre superficies de cultivo pequeñas ${ }^{4}$, con centros de producción muy próximos unos de otros y con poco esfuerzo colectivo por cuidar sanitaria y ambientalmente el recurso (Arce et al., 2016), violando la densidad aconsejable de carga del recurso en las distintas localizaciones en que se llevaban a cabo los cultivos.

La región de Magallanes, si bien presenta características particulares por su tardía incorporación a la trama productiva chilena, su modelo productivo y actores no evidencian un cambio sustancial respecto a lo observado en el resto del país. Las especies de salmónidos que se explotan en esta región son el salmón Atlántico y, en menor medida, la trucha arcoíris, concentrando el 15\% y 25\% de la producción respectiva de ambas especies en Chile.

Tabla 1. Producción de salmónidos en Chile y Magallanes, año 2017

\begin{tabular}{lccc}
\hline & Chile & Magallanes & Participación \\
\hline Salmón Atlántico & $614.173 \mathrm{t}$ & $90.561 \mathrm{t}$ & $15 \%$ \\
Trucha arcoíris & $76.960 \mathrm{t}$ & $19.251 \mathrm{t}$ & $25 \%$ \\
\hline
\end{tabular}

Fuente: SERNAPESCA.

Esta producción de salmón Atlántico le permitió a la región de Magallanes generar ingresos que, en 2017, alcanzaron valores próximos a los U\$ 500 millones $^{5}$. Por su parte, la información suministrada por el sector para toda la trama productiva reconoce la generación de aproximadamente 3100 puestos de trabajo directo y cerca de $2000^{6}$ indirectos en 2018, con 38 centros de producción activos ${ }^{7}$.

$470 \%$ más pequeña que la de Noruega (Arce et al., 2016).

5 Estimaciones propias en base a SERNAPESCA.

6 Asociación de Productores de Salmón y Trucha de Magallanes A. G.

7 Información elaborada en el marco del Proyecto FIPA 2015-42, 2017, sobre la base del Banco Cen- 
III.2. Encadenamientos productivos: características y requerimientos

La producción de salmones se realiza en tres fases: (i) la primera, de agua dulce, se inicia con la incubación de las ovas, para seguir con las etapas de alevinaje y smoltificación; (ii) la segunda fase corresponde al engorde, se realiza totalmente en el mar, iniciando con los smolts de 50/100 gramos y finaliza con la cosecha de individuos cuando alcanzan el tamaño comercial (entre 3 y 5 kg); y (iii) la tercera, el procesamiento, consiste en la faena y preparación para la comercialización.

Gráfico 1. Proceso productivo para la producción de salmón
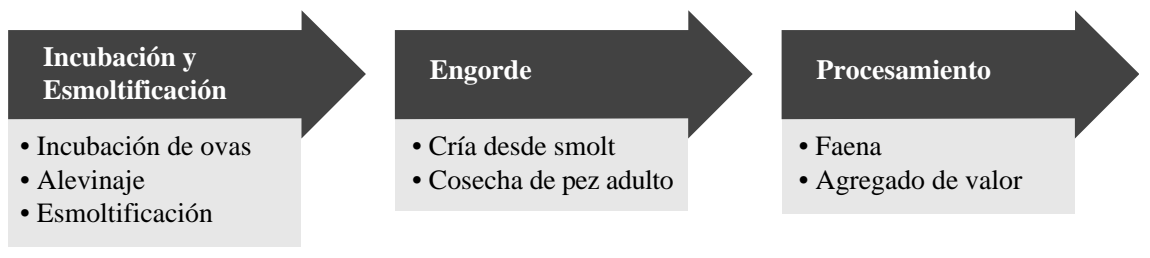

Fuente: elaboración propia.

i) Incubación y smoltificación

El proceso de incubación hasta el alevinaje (4-5 gramos) se realiza en pisciculturas en tierra, mientras que el proceso de smoltificación puede realizarse en estanques en tierra (Noruega) o en lagos (Chile). Esta diferencia radica en las diferentes regulaciones ambientales que los países mantienen con relación a la utilización de espejos de agua dulce naturales para el desarrollo de la acuicultura (más permisivas en Chile que en Noruega).

Existe en la producción de ovas un componente biotecnológico importante asociado al mejoramiento genético y la sanidad. En general, Noruega y Escocia son dos referentes, con participación pública y privada en los sistemas de innovación y procesos de inversión asociados ${ }^{8}$.

tral, SERNAPESCA y el INE, estimaba el empleo directo generado por toda la trama en Magallanes en 2432 puestos de trabajo, y en 1563 el empleo indirecto, para 2015.

8 Diagnóstico Económico y Social de la Acuicultura en Chile. FIP N. 2002-24, Coquimbo, 2005 (446-450). 
ii) Engorde

El sistema de engorde requiere de jaulas flotantes para el desarrollo del smolt hasta su etapa adulta, proceso que puede demandar entre 12 y 20 meses en aguas con las características de esta región ${ }^{9}$. Las jaulas habitualmente están dispuestas en grupos de 10 a 20 con capacidad de albergar entre 25 y 180 toneladas cada una, dependiendo de las dimensiones y densidades de carga.

Las principales operaciones son la alimentación, manejo sanitario, monitoreo del crecimiento de los peces, mantención de las estructuras y, finalmente, la cosecha, que se produce cuando los peses han alcanzado el tamaño comercial de 3 a $5 \mathrm{~kg}$. Los animales son sacrificados y colocados en cajas con hielo para ser procesados en plantas, o bien se transportan vivos en wellboats (embarcaciones para este fin) hasta las plantas de faena y procesamiento.

Durante la etapa de crecimiento y engorde en el mar las redes que conforman la jaula deben cambiarse por redes de malla mayor, a medida que los peces van aumentando de peso y talla. Esos recambios se producen aproximadamente cada 5-6 meses. Los insumos básicos en esta etapa son los alimentos balanceados, cuyo factor de conversión en Chile se calcula en un promedio de 1.3 kilos de alimento por cada kilo de carne, pudiendo variar por temperatura, condiciones del medio marino y tipo de alimento. La composición de este alimento debe contener una dieta balanceada, rica en proteínas y ácidos grasos, complementada con carbohidratos, vitaminas y minerales. Esta composición se complementa con pigmentos que garantizan la coloración rojiza de la carne ${ }^{10}$.

El otro componente clave en la producción de salmónidos a escala intensiva son los antibióticos utilizados para combatir enfermedades.

iii) Procesamiento

Esta etapa puede iniciar con la matanza del animal en caso de recibirlo vivo, para luego proceder a su faenamiento, es decir, corte de agallas, descabezamiento y eviscerado. El producto final es el animal entero o descabezado (HG), que puede comercializarse refrigerado o congelado.

9 En aguas más cálidas el proceso podría extenderse por lo menos cuatro meses más.

10 Diagnóstico Económico y Social de la Acuicultura en Chile. FIP N. ${ }^{\circ}$ 2002-24, Coquimbo, 2005 (446-450). 
Tabla 2. Inversiones, empleo y servicios requeridos por fase productiva

\begin{tabular}{lll} 
Fase & $\begin{array}{l}\text { Infraestructura y requerimientos } \\
\text { tecnológicos }\end{array}$ & Empleo y servicios regulares \\
\hline $\begin{array}{lll}\text { Alevinaje y } \\
\text { smoltificación }\end{array}$ & $\begin{array}{l}\text { Estanques y pisciculturas, } \\
\text { laboratorios de diagnóstico, } \\
\text { equipamiento para el trata- } \\
\text { miento de efluentes, dispensa- } \\
\text { dor automático de alimentos. }\end{array}$ & $\begin{array}{l}\text { Servicios especializados en } \\
\text { biotecnología, personal para } \\
\text { servicios de desinfección de } \\
\text { camiones, retiro de mortan- } \\
\text { dad, consultorías ambienta- } \\
\text { les, análisis de diagnóstico, } \\
\text { transporte de ovas, personal } \\
\end{array}$ \\
& $\begin{array}{l}\text { y alimento, control de plagas, } \\
\text { mantenimiento del equipa- } \\
\text { miento, entre otros. }\end{array}$
\end{tabular}

Engorde Jaulas, redes, oficinas, cone- Especialista en alimentación, xión terrestre, redes eléctricas, especialista en manejo sanidispensador de alimentos tario, especialista en buceo automático, máquina de cose- y manejo de redes, operario cha, pileta de anestesia o kno- de servicios generales para queadora o wellboat, muelle mantención de instalaciones, especial, tuberías y bombas. seguridad y vigilancia, especialista en manejo de cultivos, especialista en cosecha, especialista en operaciones de wellboat, servicios de transporte terrestre.

$\begin{array}{lll}\begin{array}{l}\text { Procesa- } \\ \text { miento }\end{array} & \begin{array}{l}\text { Redes eléctricas. Planta de } \\ \text { procesamiento y equipos de } \\ \text { refrigeración. }\end{array} & \begin{array}{l}\text { Higiene, embalaje, transporte } \\ \text { de personal y peces, labora- } \\ \text { torio de diagnóstico, consul- } \\ \text { torías ambientales, control y } \\ \text { planificación de la produc- } \\ \text { ción, fileteador, entre otros. }\end{array} \\ \end{array}$

Fuente: elaboración propia. 
Una instancia de mayor agregación de valor sería el desarrollo de las operaciones necesarias para la obtención de cortes de salmón. Es la etapa que mayor mano de obra requiere, dado que esos cortes deben realizarse de manera manual, requiriendo algunos trabajadores con habilidades específicas, como el fileteador. Los productos obtenidos son los diversos filetes y trozos de salmón.

Esta etapa concluye con la colocación de los peces en los envases definitivos para su venta frescos o congelados, siendo transportados por vía aérea o marítima hasta los destinos comerciales.

Cabe señalar que estas tres etapas pueden integrarse verticalmente en una única empresa, cerrando el círculo productivo, o lo que es más habitual, sobre todo en los inicios de la actividad, comenzar con la explotación de engorde en el mar, adquiriendo externamente los smolts y contratando el procesamiento como un servicio externo ${ }^{11}$.

En los inicios de la actividad en la región de Magallanes, la mayor parte de estos servicios, incluso la mano de obra especializada, provenía de las regiones centrales del país. Con el paso del tiempo, muchos de estos servicios y recursos se fueron localizando en el territorio.

\section{III.3. Marco normativo e institucional}

El deterioro de las condiciones sanitarias de la salmonicultura chilena, la sucesión de diversas crisis ambientales y el aumento progresivo en las áreas y volúmenes de producción obligaron a una continua readaptación de los mecanismos de regulación.

Así, estas problemáticas emergieron como factores de importancia a la luz de la profunda crisis sanitaria y ambiental que debió enfrentar la industria salmonera chilena en los años 2000 (Katz \& Araya, 2015), reforzando el carácter reactivo que históricamente ha caracterizado la función reguladora del Estado en la materia (Paredes \& Martínez, 2018). Estos hechos trasparentaron la existencia de un sector público poco involucrado en controlar al sector privado, aun cuando la actividad se basa en el uso bienes de propiedad colectiva (franja costera, ríos o lagos).

11 En general, cuando las plantas de procesamiento no están integradas verticalmente al engorde, estas suelen contratarse como un servicio, sin transferir la propiedad del animal. 
A raíz de la crisis de 2007/2008, ocurrida por la epidemia de virus ISA (Anemia Infecciosa del Salmón) que se propagó rápidamente por los centros de cultivo de la región de Los Lagos ${ }^{12}$, el gobierno chileno creó un nuevo espacio públicoprivado de concertación, denominado la Mesa del Salmón ${ }^{13}$, con el propósito de rescatar la industria, centrando su trabajo en mejorar las condiciones para el cultivo de salmónidos (Katz et al., 2011).

Si bien Chile ha avanzado en la construcción de un marco institucional y normativo orientados a acompañar el desarrollo de la actividad (ver anexo: tablas 1 y 2), estos no han sido suficientes para evitar tragedias ambientales, problemas económicos y conflictos sociales.

III.4. Problemas ambientales, económicos y sociales del caso chileno

El análisis del caso chileno pone en evidencia la importancia de las cuestiones sanitarias y del impacto ambiental que puede generar la actividad, más aún en territorios donde la explotación se realiza a partir de especies exóticas.

Uno de los principales problemas para la actividad es la permanente presencia de enfermedades, como SRS y caligidosis, con importantes consecuencias económicas. En particular, el virus ISA sumió en el año 2007 a la industria del salmón en una profunda crisis, provocando una pérdida cercana al 25\% de la producción, el cierre masivo de centros de cultivos y la pérdida de alrededor de 20000 puestos de trabajo directos e indirectos, afectando severamente la economía de la Región de Chiloé ${ }^{14}$. Este virus se ha detectado recientemente en la región de Magallanes ${ }^{15}$.

Como consecuencia de los cambios regulatorios que se dieron en respuesta a estas severas crisis, los costos de producción se incrementaron notablemente, pasando de u\$ 2/kg a más de u\$ 4/kg entre 2007 y 2014. En este sentido, tanto el

12 Las firmas debieron sacrificar millones de peces, viéndose impedidas de cubrir sus deudas con el sistema bancario, el que se negó a refinanciar nuevas campañas de siembra y las firmas perdieron buena parte de su valor de mercado al tener que sacrificar la biomasa en cultivo (Katz \& Araya, 2015 y Arce et al., 2016). El 60\% de los centros de cultivo dejaron de producir, reduciendo el empleo directo en aproximadamente 25000 puestos de trabajo (Arce et al., 2016).

13 Integrada por la Subsecretaría de Pesca (SUBPESCA), el Servicio Nacional de Pesca y Acuicultura (SERNAPESCA), la ex Comisión Nacional del Medio Ambiente (CONAMA), la Subsecretaría de Marina, la CORFO, la Fundación Chile y expertos a título personal. http://www.aqua.cl/2019/11/25/sernapesca-confirmo-caso-de-virus-isa-en-magallanes/ 
sector privado como representantes del sector académico han señalado la construcción de un abordaje regulatorio inadecuado que carga con costos excesivos a la actividad, sin propiciar los efectos buscados ${ }^{16}$.

Vale decir que las tasas de mortalidad comenzaron a moderarse en los últimos años. Según el Reporte de Sustentabilidad publicado por SalmonChile, en 2013 la mortandad promedio fue de 7.7\%, de 6.5\% en 2014, en 2015 disminuyó hasta un 5\% y para 2016 se estimaban en un $3 \%{ }^{17}$. No obstante, las pérdidas estimadas alcanzarían los u\$ 800 millones anuales ${ }^{18}$, existiendo denuncias penales por ocultamiento de daño ambiental vía manejo fraudulento de las declaraciones juradas en una de las principales explotaciones “modelo” de la Región de Magallanes ${ }^{19}$.

Desde la perspectiva ambiental, los principales impactos de la salmonicultura en la zona costera son: (i) eutrificación ${ }^{20}$ de la columna de agua como consecuencia de la acumulación de residuos sólidos y líquidos en el fondo marino, derivada de los alimentos no consumidos, fecas y mortalidad de los peces; (ii) transmisión de enfermedades a la fauna silvestre; (iii) uso de antibióticos con consecuencias sobre agua y suelo; (iv) fugas masivas, cuyos principales efectos son la disminución de las especies nativas a través de la depredación, la competencia y la transmisión de enfermedades, además del asilvestramiento de los salmones en la zona; (v) presión sobre especies pesqueras silvestres, utilizadas para harina y aceite de pescado para alimentar a los salmónidos; (vi) interacciones negativas directas e indirectas con mamíferos marinos y aves, algunas de estas especies con estados de conservación delicados ${ }^{21}$; y (vii) desechos industriales como jaulas, plásticos, boyas, cabos, entre otros. Esto se suma a las transformaciones visuales de paisajes naturales.

La falta de conocimiento profundo sobre los efectos ecosistémicos de estas situaciones impide cuantificar de manera precisa los costos asociados al desarrollo

16 http://www.aqua.cl/columnas/los-efectos-de-la-normativa-sanitaria-en-la-salmonicultura-nacional/

17 Aqua, 20/03/2017: En 2016 mejora la salud de la salmonicultura.

18 http://www.aqua.cl/2016/11/30/aqua-en-ruta-hasta-donde-puede-llegar-la-expansion-de-la-salmonicultura-en-magallanes/\#

19 https://www.elmostrador.cl/noticias/pais/2019/07/09/gobierno-denuncia-a-salmonera-nova-australpor-manipulacion-y-ocultar-dano-ambiental-ante-el-ministerio-publico/

20 La eutrificación o eutrofización produce un crecimiento masivo de las microalgas que tiñe el agua de verde. Este color provoca que la luz solar no entre a las capas más bajas del agua por lo que las algas de ese nivel no reciban luz para realizar la fotosíntesis, lo que conlleva a la muerte de las algas. La muerte de las algas genera un aporte extra de materia orgánica por lo que el lugar se vuelve putrefacto y un ambiente reductor (esto significa un ambiente bajo en oxígeno).

21 Foro para la Conservación del Mar Patagónico y Áreas de Influencia, 2018. 
de la actividad. Resulta fundamental realizar estudios de carga serios que permitan contar con más información a este respecto.

A la luz de los impactos sobre el ecosistema marino y los paisajes naturales, ha proliferado la manifestación de organizaciones y movimientos sociales en Chile, que fueron incrementando su presencia ante cada catástrofe evidenciada, sumando actores de instituciones variadas (académicas, científicas, ambientalistas), siendo su principal eje de debate el uso de los recursos y su regulación. En marzo de 2019, las manifestaciones en Puerto Williams frente a la visita de los reyes de Noruega pusieron en evidencia las tensiones sociales suscitadas por la actividad en la región de Magallanes, principalmente frente a la posibilidad de ampliar la misma en el canal Beagle22.

\section{DISCUSIÓN Y REFLEXIÓN SOBRE EL CASO DE LA COSTA ARGEN- TINA DEL CANAL BEAGLE}

El caso de la región de Magallanes permite reflexionar sobre la posible instalación de la actividad productiva de salmones intensiva a gran escala en la costa argentina del canal Beagle, la cual se asocia, en primera instancia, a la disponibilidad del recurso marino para el desarrollo de la fase de engorde.

\section{IV.1. Encadenamientos. Características y requerimientos}

Por la forma en que se estructura este sector, es habitual que las empresas productoras compren los smolts para su engorde, desarrollen esta fase y luego contraten los servicios de procesamiento. Si bien estas etapas pueden estar integradas verticalmente, no es lo esperable en el período inicial de radicación de la actividad.

Entre el conjunto de actividades directa e indirectamente asociadas a la industria del salmón, se identifican algunos rasgos relevantes. En primer lugar, las actividades intensivas en $\mathrm{I}+\mathrm{D}$ aparecen asociadas principalmente a las primeras fases (producción de alevines, smoltificación y engorde), donde son relevantes aspectos biológicos, genéticos, inmunológicos y de salud animal. En estos campos son importantes los desarrollos de genética animal, vacunas, antibióticos, desarro-

22 https://m.elmostrador.cl/agenda-pais/2019/03/27/por-que-se-cuestiona-que-noruega-siga-trayendosalmoneras-a-nuestro-territorio/ 
llo de alimentos, y diversos aspectos vinculados al cuidado del medio ambiente (Zanlungo et al., 2015).

En segundo lugar, existe una trama de servicios especializados (mantenimiento de redes, servicios de buceo, estudios de genética, servicios veterinarios, entre otros) que en el caso de Chile se han desarrollado principalmente en el entorno de la región central, aunque con el paso del tiempo, tal como fuera mencionado precedentemente, se han ido generando algunos de estos servicios también en la región de Magallanes. Sin embargo, por su especificidad, no es esperable que los mismos estén disponibles en el territorio argentino bajo análisis.

En tercer lugar, tanto el proceso de smoltificación, cuando se realiza en espejos de agua natural como suele ser el caso en Chile, como la fase de engorde, requieren condiciones naturales especiales, constituyendo su disponibilidad la principal condición para el desarrollo de la actividad. Estas fases son las que concentran los mayores costos y desafíos desde el punto de vista ambiental y donde se podría insertar claramente la provincia argentina de Tierra del Fuego.

En cuarto lugar, la fase de procesamiento concentra la mayor generación de puestos de trabajo en actividades de relativamente baja complejidad. Si bien podría plantearse la instalación de plantas de procesamiento en las inmediaciones de Ushuaia, al analizar la situación del sector en la parte chilena de Tierra del Fuego, se puede especular con una potencial integración productiva. Allí existen empresas radicadas en las proximidades de la localidad de Porvenir, ubicada al noroeste de la isla Grande de Tierra del Fuego, donde se están realizando inversiones que apuntan a integrar verticalmente las etapas de engorde, con la producción de smolts y el procesamiento.

En este sentido la empresa Nova Austral está realizando inversiones por aproximadamente U\$ 40 millones para instalar una piscicultura con una capacidad productiva de 12 millones de smolts al año ${ }^{23}$. Mientras tanto, el abastecimiento de smolts se realiza por vía marítima, principalmente desde la región de Los Lagos y Aysen. La misma empresa cuenta con una planta con capacidad para procesar 40000 toneladas, generando 600 puestos de trabajo directo, proyectando inversiones que le permitirán aumentar su capacidad en 15000 toneladas en los próximos años ${ }^{24}$. Considerando que además de esta empresa existen en la región de Magallanes otras cinco dedicadas a la acuicultura de salmónidos, parece razonable

23 Fuente: www.aqua.cl

24 Fuente: www.mundoacuicola.cl 
pensar en un modelo integrado regionalmente, que aproveche principalmente las condiciones productivas que ofrece el recurso marino en el canal Beagle para el engorde de los animales, quedando la eventual posibilidad de procesar en torno a la ciudad de Ushuaia supeditada a un análisis de costos, donde la mano de obra calificada resulte una variable limitante.

Bajo estas consideraciones, y teniendo en cuenta las condiciones existentes en el territorio fueguino, el aporte a la cadena global de valor se limitaría a la explotación de sus recursos naturales, a través del desarrollo de la fase de engorde, recibiendo la mayor parte de los insumos y servicios desde el exterior, principalmente del país vecino.

Para poder hacer estimaciones de producción, empleo, inversiones e ingresos, se ha recurrido a un relevamiento realizado en 1993, única referencia disponible para analizar el potencial de la acuicultura en Tierra del Fuego argentina (Quirós et al., 1993) y a entrevistas con informantes clave. En el estudio mencionado se identificaron nueve sitios marinos en el canal Beagle y litoral Atlántico Sur con potencial para el desarrollo de la acuicultura con modalidad en jaulas suspendidas en sistema intensivo, estableciéndose una capacidad de sustentación de 20000 toneladas anuales. Si bien durante 2018 el gobierno de Tierra del Fuego ha realizado un estudio con la intención de analizar los sitios y el potencial de explotación en el marco de un acuerdo entre el gobierno provincial de Tierra del Fuego y la Fundación Argentina para la Promoción de Inversiones y Comercio Internacional, los resultados aún no han sido publicados. Por otro lado, las fuentes recientemente consultadas estiman dicha capacidad en valores superiores, posiblemente un poco más de 30000 toneladas al año.

En este marco, se estima en la etapa de engorde que para una producción de 20.000 a 30.000 toneladas/año, se requeriría la instalación de 120 a 180 jaulas de gran porte. A partir de estos datos, se puede estimar el empleo potencial. Para ello se utilizará como parámetro información relevada por Dresdner et al. (2017), sobre la base de datos de SERNAPESCA para 2014 en la región de Magallanes, donde se generaron 278 puestos de trabajo directo en centros de cultivo, a razón de 2.61 puestos por tonelada producida. Por otro lado, el trabajo de Chávez y Zuñiga (2011) establece que la empresa Nova Austral (una de las más importantes en Magallanes) requiere para operar de 15 personas por centro de producción, entre ellos 3 profesionales para cubrir los roles de encargado de la concesión, encargado de medio ambiente y encargado de gestión. En ese mismo sentido, entrevistas a informantes clave indicarían la necesidad de contar con un jefe de centro y dos asistentes, de 
perfiles ingenieros o biólogo especialista en acuicultura; 4 técnicos y alrededor de 6 operarios de baja calificación.

Considerando estas referencias, y de acuerdo con el potencial de explotación estimado para la costa argentina del canal Beagle, se estima un potencial de generación de empleo directo de aproximadamente 75 puestos de trabajo, asociados directamente a la fase de engorde en aproximadamente 5 centros de cultivo.

Por su parte, dentro de las inversiones productivas, la más importante consiste en las plantas de engorde, incluyendo jaulas y redes, con un costo aproximado de u\$ 60 millones.

Cuadro 3. Inversiones productivas para una capacidad estimada en 30000 toneladas anuales

\begin{tabular}{cccc}
\hline Concepto & Costo Unitario & Cantidad & Costo total \\
\hline $\begin{array}{c}\text { Planta de engorde capacidad } \\
6000 \text { toneladas } \\
\text { (incluye jaulas y redes) }\end{array}$ & $\mathrm{u} \$ 12$ millones & 5 & u\$ 60 millones \\
\hline
\end{tabular}

Fuente: elaboración propia sobre la base de informantes del sector.

Finalmente, con esta capacidad productiva se estima un nivel de facturación anual cercano a los u\$ 100 millones.

IV.2. Marco normativo e institucional

En la provincia de Tierra del Fuego, la actividad acuícola se encuentra regulada por el Ministerio de Agricultura, Ganadería y Pesca, en tanto Autoridad de Aplicación de la Ley Provincial de Pesca 244/95, modificada por la Ley 537/01 y reglamentada mediante el Decreto provincial 670/07 (anexo: tabla 4).

Esta normativa refiere a: a) las áreas de cultivo y especies a determinar conjuntamente con los organismos encargados de los usos alternativos de los terrenos, agua y medio ambiente; b) las autorizaciones, concesiones y licitaciones, su duración, renovación, caducidad, infracciones, requisitos mínimos a cumplimentar y posibilidad de transferencia; c) canon y d) el requisito de inscripción en el Registro Provincial que lleva la Dirección de Pesca y Acuicultura, como así también en el 
Registro Nacional de Acuicultura. Si bien el Decreto Reglamentario 670/07 menciona explícitamente la salmonicultura, lo hace refiriéndose a los proyectos que se desarrollen en agua dulce y no regula sobre aquellos que potencialmente podrían instalarse en aguas del canal Beagle.

En el año 2017, Tierra del Fuego adhirió a la Ley Nacional de Desarrollo Sustentable del Sector Acuícola 27231/15 por medio de la Ley Provincial 1168, reglamentada mediante el Decreto 2215/17, en la que se explicitan las exenciones impositivas (parciales y transitorias en el impuesto sobre Ingresos Brutos y la tasa de sellado) que serán consideradas para promover el desarrollo de la actividad.

Esta ley habilita la posibilidad de introducir especies exóticas, siempre que se cuente con el permiso de introducción

extendido por las autoridades competentes, nacional o provincial, al efecto de la aprobación de una solicitud emitida por un interesado para importar individuos y/o subproductos de una especie de organismo acuático, de carácter exótico que se desee introducir al territorio argentino (Artículo 5, inciso k de la Ley Nacional 27231).

Dada la adhesión en todos sus términos, resulta importante considerar algunos elementos de la normativa nacional, los que tienen plena vigencia para el territorio provincial. Así se destaca la mayor especificidad de la normativa nacional en cuanto a la determinación de los sistemas de producción acuícola, los que podrán ser "planificados como extensivos, semi-intensivos e intensivos, según la densidad utilizada en cultivo y el grado de tecnología aplicado", pudiendo ser ejecutados a “cielo abierto” o “en encierro” (Artículo 6 de la Ley Nacional 27231).

Del mismo modo, la normativa nacional establece responsabilidades provinciales (y nacionales) respecto al "aprovechamiento sustentable de sus recursos acuícolas, la conservación del medio, la restauración del mismo de ser necesario y la protección de aquellos ecosistemas en los que se realicen cultivos de peces u otros organismos acuáticos”, por lo que las autoridades provinciales (o nacionales) deberán proceden a la

determinación de la 'capacidad de carga' o ‘capacidad de soporte' de los mismos; con el objeto de sustentar las potenciales unidades de cultivo. De esta forma, los sistemas acuáticos públicos, naturales o artificiales, sometidos a producción acuícola se mantendrán, en lo posible, ecológicamente 
sustentables en el tiempo, soportando producciones acordes a sus características biológicas (Artículo 7 de la Ley Nacional 27231).

En este sentido se establecen principios rectores que deberían guiar la elaboración de normativa reglamentaria que de manera precisa estipulen las reglas de explotación y conservación de los espacios naturales.

Por último, vale decir que la Ley Nacional prevé la implementación de "programas de vigilancia epidemiológica, desarrollándolos con la colaboración de las provincias y otras entidades involucradas”. Asimismo, el Servicio Nacional de Seguridad y Calidad Agroalimentaria (SENASA), deberá desarrollar

dentro de su Plan Nacional de Sanidad de Animales Acuáticos, lo referido a la prevención y control de contingencias y de monitoreo, así como normativas que, por las características propias de las especies explotadas, sean requeridas para el mantenimiento de la actividad sustentable en todas las cuencas acuícolas del territorio (Artículo 8 de la Ley Nacional 27231).

Con todo lo expuesto, el marco regulatorio provincial parecería mostrar varias debilidades. En primer lugar, existe un escaso miramiento de la salmonicultura dentro del entramado regulatorio del territorio, lo que limita las posibilidades de establecer consideraciones específicas respecto a las acciones que deberían llevarse a cabo para garantizar un desarrollo sustentable de la actividad en el largo plazo.

En segundo lugar, la independencia en cuanto a la información requerida para fiscalizar y controlar los riesgos potenciales de la actividad, principalmente los relativos a enfermedades, adolece de falencias en tanto solo explicita la denuncia obligatoria por parte del acuicultor, no estableciendo en la normativa general o reglamentaciones específicas posteriores otras actividades de contralor por parte de la Autoridad de Aplicación o, en tal caso, la modalidad y frecuencia de su accionar ${ }^{25}$.

En tercer lugar, y muy relacionadas a la primera observación, las escasas acciones consideradas obedecen más al criterio correctivo que preventivo, pensadas a priori para actividades acuícolas de pequeña escala, que nada tienen que ver con

25 Bajo la normativa vigente es obligatoria la denuncia por parte del acuicultor a la Autoridad de Aplicación ante "la presencia o presunta presencia de enfermedades a fin de erradicarlas y prevenir su propagación”; quedando explicitado que en caso de infracción "se podrá cancelar la concesión sin perjuicio de las sanciones que prevé la presente Ley y el reclamo por los daños y perjuicios que dicha omisión haya ocasionado” (Art 37, Ley Provincial 244). 
una producción intensiva de salmones. Al respecto vale decir que, si bien la normativa nacional a la que adhirió la provincia recientemente establece medidas de vigilancia epidemiológica, prevención y control de contingencias, estas son aún de carácter general, tanto en la dimensión biológica (requerimientos vinculados a los salmones), como territorial (requerimientos específicos de la actividad en estas latitudes).

Para avanzar en el análisis cabe preguntarse acerca de las capacidades institucionales, entendidas como la cantidad y calidad de recursos (humanos y de infraestructura) de los que dispone el gobierno provincial, en su carácter de Autoridad de Aplicación para la fiscalización y el control de las actividades acuícolas a desarrollarse en la provincia.

De acuerdo con fuentes oficiales de la Dirección General de Desarrollo Pesquero y Acuícola ${ }^{26}$, el personal de planta ascendería a 12 agentes ${ }^{27}, 5$ de ellos abocados específicamente a actividades de fiscalización y control pesquero, acuícola y marisquero (embarcado y no embarcado) de toda la provincia. Por otro lado, los niveles actuales de remuneración del personal dificultan la posibilidad de acceder a recursos humanos calificados.

Consultado sobre los recursos necesarios ${ }^{28}$ para cubrir las actividades de fiscalización y control ante el potencial desarrollo de la actividad salmonera intensiva, la fuente oficial referenció en primera instancia las dificultades logísticas vinculadas a los lugares potenciales de instalación de los centros de cultivos, incrementando los requerimientos de recursos.

No solo se necesitaría disponer de mayor cantidad de personal capacitado (mínimos 30 personas), sino que estos deberían tener movilidad y permanencia en zonas cercanas a los mencionados centros. Esto requeriría el establecimiento de vivien-

26 Dependiente de la Subsecretaría de Pesca del Ministerio de Agricultura, Ganadería y Pesca de la Provincia de Tierra del Fuego. De esta Dirección General se desprende la Dirección de Desarrollo Productiva (abocada a las actividades acuícolas hoy desarrolladas en la provincia) y la Dirección de Investigaciones Pesqueras (abocada a las actividades de contralor de la pesca de altura).

27 Incluye directores, personal administrativo y personal afectado a la fiscalización y control pesquero, acuícola y marisquero, tanto embarcado como no embarcado. No incluye a los observadores a bordo vinculados a las actividades de pesca de altura, por desconocimiento del informante.

28 Respecto a la infraestructura disponible para las actividades de fiscalización y control, la fuente consultada informa la existencia de dos botes semirrígidos, tres camionetas, dos vehículos UTV para el recorrido en playa y la existencia del Laboratorio Ambiental, emplazo en la piscicultura provincial, que a pesar de continuar manteniendo esta denominación, su tarea se focaliza en el análisis de toxinas marinas (marea roja) para mejillones, actuando de acuerdo al Protocolo de SENASA (autoridad sanitaria). 
das, con sus respectivos servicios básicos, además de infraestructura pública para acoger al personal involucrado y realizar diferentes tareas (entre ellas análisis de agua, epidemiológico, debiendo adquirirse también el material de laboratorio necesario).

Asimismo, debido a la lejanía del puerto de atraque más cercano (Almanza), se requeriría de la construcción de un embarcadero en zona, el que debería permitir el amarre permanente de una embarcación de la Autoridad de Aplicación.

A lo antes mencionado debe agregarse que las grandes extensiones de espacios y recursos naturales generan un gran desafío para su resguardo y control, quedando en evidencia las limitaciones institucionales presentes en la provincia para hacer frente a esto.

Según informantes de las distintas áreas involucradas, estas falencias están relacionadas con la baja cantidad de personal disponible y la escasa disponibilidad de recursos económicos, lo que dificulta la adquisición de material de trabajo y el mantenimiento de los existentes. Algunas fuentes resaltan la falta de profesionalismo del personal de control involucrado, lo que puede afectar la eficacia de las acciones.

Finalmente, cobra relevancia el análisis de las instituciones de I+D+i que actúan en el territorio. A nivel local, el Centro Austral de Investigaciones Científicas (CADIC-CONICET), la Universidad Tecnológica Nacional (UTN) y la Universidad Nacional de Tierra del Fuego son las principales instituciones que podrían actuar de referencia para el sector.

A pesar de los esfuerzos formativos y de investigación inicial, la realidad territorial muestra una débil relación entre el sector productivo y el científico tecnológico local. Según se evidencia de las entrevistas realizadas, instituciones como CADIC, con capacidad científico-tecnológica para acompañar las necesidades que requiere el sector, ha expresado la decisión de no involucrarse a raíz del esquema productivo que se plantea en este proyecto y los potenciales riesgos asociados.

IV.3. Riesgos ambientales, conflicto económico y social del caso argentino en el canal Beagle

Si bien cada territorio representa una realidad política, social, institucional y ecosistémica diferente, por lo que no cabe esperar que la evolución de un mismo sector económico discurra de manera similar, las lecciones aprendidas a la luz de la experiencia chilena ponen en foco los riesgos involucrados en el desarrollo de la actividad. 
Bajo estas consideraciones, los impactos ambientales dependerán de las cargas, ordenamiento y capacidad del Estado para regular y monitorear la actividad. Sin embargo, en ningún caso podrán evitarse los deterioros del suelo marino y la calidad del agua, al igual que la mayor parte de las consecuencias señaladas para el caso chileno, ya que son parte constituyente del modelo productivo.

Del mismo modo, los riesgos sanitarios y los impactos por las fugas dependerán fuertemente de las prácticas productivas y esencialmente de las regulaciones y capacidad de fiscalización.

En este sentido, el marco regulatorio de Tierra del Fuego, las capacidades institucionales y las experiencias vividas en relación con la capacidad efectiva de fiscalización del Estado ${ }^{29}$ posicionan al territorio provincial en un escenario de fuerte debilidad y alto riesgo.

Por otro lado, los lugares que potencialmente podrían ser ocupados por estas explotaciones en las costas del Canal son compartidos por pequeñas explotaciones de pesca artesanal y acuicultura, cuya producción se orienta fuertemente al consumo local, enriqueciendo la oferta gastronómica orientada al turismo. Este tipo de producciones (moluscos, mejillones y centolla) depende fuertemente de la calidad del agua.

Algo similar ocurre con la actividad turística que, con 5258 fuentes de empleo directo (SIPA, 2018), se constituye en la principal actividad productiva de la zona sur de la provincia. Considerando que Tierra del Fuego se posiciona como un territorio prístino y lejano en el "fin del mundo", que ofrece una experiencia asociada a lo salvaje (Vereda \& Jensen, 2014), la potencial instalación de jaulas para el engorde de salmones atentaría contra la calidad del recurso paisajístico, con un impacto directo sobre las navegaciones en el Canal.

$\mathrm{Al}$ igual que en el territorio chileno, la potencial instalación de la actividad salmonera ha despertado la crítica social, materializada en múltiples manifestaciones que hacen eje en los potenciales impactos negativos de una industria de esta naturaleza. Vale la pena destacar el movimiento "NO a las salmoneras en el Canal Beagle”, que nuclea a distintas ONG, sociedad civil, comunidad científica, representantes de la actividad turística, entre otros actores, que ha llevado adelante

29 Problemas de fiscalización del Estado en los sectores vinculados a la explotación de recursos naturales. Esto a raíz de, entre otras cuestiones, la falta de recursos humanos y materiales para abordar la amplitud y diversidad del territorio. 
diversas manifestaciones expresando el rechazo de la actividad. Entre ellas se resaltan cine debate, charlas abiertas, manifestaciones públicas, conferencia de prensa, así como diversas manifestaciones públicas que pretendieron dar visibilidad al conflicto. Expresiones de similar naturaleza se observaron simultáneamente en la ciudad de Puerto Williams, perteneciente a la costa chilena del Canal, en el marco de actividades conjuntas impulsadas por el mencionado movimiento.

Asimismo, el Foro para la Conservación del Mar Patagónico y Áreas de Influencia ${ }^{30}$, que agrupa a 23 ONG ambientalistas, expresó su preocupación por los riesgos que la salmonicultura podría causar, emitiendo una declaración de rechazo a la actividad, bajo el lema compartido "Canal Beagle Sin Salmoneras”31.

Ambos colectivos sociales reclaman la sanción de una ley prohibitiva del ejercicio de este tipo de actividades por sus potenciales impactos sobre el paisaje, el ejercicio de otras actividades productivas (pesca artesanal, acuicultura, turismo) y el ecosistema marino ${ }^{32}$.

\section{CONCLUSIONES}

Las lecciones aprendidas a la luz de la experiencia chilena y la consideración de las capacidades existentes en el territorio bajo análisis se tornan de suma utilidad para analizar la potencialidad de la actividad en las costas argentinas del canal Beagle.

El caso chileno presenta características de un modelo exitoso para ofrecer crecimiento económico y diversificar la estructura productiva, pero que no ha dado respuestas satisfactorias para transitar un sendero de desarrollo sustentable.

Esta experiencia deja en claro la dificultad de diseñar un marco regulatorio y construir un aparato institucional capaz de resolver los problemas asociados al uso de recursos comunes, lo que ha perpetuado los conflictos sociales en el territorio, magnificados por las catástrofes ambientales asociadas al desarrollo de la actividad.

El rol del Estado no debe limitarse a establecer las reglas de juego, sino que debe ser capaz de ejercer un control efectivo y garantizar los esfuerzos tecno-

\footnotetext{
30 https://marpatagonico.org/

31 https://marpatagonico.org/descargas/declaracion-regional-salmonicultura-canal-beagle.pdf

32 Fuente https://www.diarioprensa.com.ar/no-a-las-salmoneras-en-el-canal-beagle/
} 
lógicos direccionados a la sustentabilidad de la actividad. Las posibilidades reales de apropiación de rentas, así como la disminución de daños y riesgos ambientales involucrados en el desarrollo de la actividad, guardan estrecha relación con esta capacidad del Estado.

El caso argentino parece presentar riesgos adicionales en tanto que la actividad se plantea en términos netamente extractivos. Con esto quiere decirse que los potenciales beneficios del caso analizado quedan supeditados a la escasa capacidad del territorio de generar encadenamientos en los eslabones de mayor valor agregado. A priori, el contexto permite inferir que las actividades se desarrollarían en la etapa de engorde para aprovechar la disponibilidad de un recurso natural, respondiendo a las características de un enclave extractivista sin mayores oportunidades, al menos en el corto plazo, de aprovechamiento de recursos locales de otra naturaleza (conocimiento y mano de obra especializados).

Del mismo modo, la información disponible, no permite avizorar un desarrollo tecnológico y de capacidades locales similar al ocurrido en Chile. La falta de una estrategia integral, sumada a la baja capacidad de gestión en las áreas de control provincial, así como la no incorporación de actores clave como CADIC (que posee capacidades pero decide no formar parte del proyecto), son indicios de esta situación. 


\section{ANEXO}

Tabla 1. Institucionalidad involucrada con el desarrollo de la acuicultura de salmones en Chile

Institución Rol

Corporación de Fomento Financiamiento. Integrante de la Mesa de Salmón.

para la Producción

(CORFO)

$\begin{array}{ll} & \begin{array}{l}\text { Proteger y mejorar las condiciones ambientales, genéticas y } \\ \text { geográficas de los productos silvoagropecuarios. Controla }\end{array} \\ \text { Servicio Agrícola y } & \begin{array}{l}\text { la calidad de los alimentos y medicamentos para animales, } \\ \text { de modo que no afecten negativamente al animal o a su } \\ \text { Ganadero (SAG) }\end{array} \\ \text { consumidor final. }\end{array}$

Fomentar la creación de nuevas empresas, al demostrar la

Fundación Chile factibilidad y atractivos iniciales de la salmonicultura chilena. Integrante de la Mesa de Salmón.

(SUBPESCA)
Principal organismo regulador. Proponer las normas de protección, control y aprovechamiento racional de los recursos hidrobiológicos y de su medio, definiendo por resolución las densidades de cultivo de salmónidos. Integrante de la Mesa de Salmón.
Servicio Nacional de

Pesca y Acuicultura (SERNAPESCA)
Principal organismo fiscalizador. Ejecutar la política pesquera nacional y fiscalizar su cumplimiento, y en especial, velar por la debida aplicación de las normas legales y reglamentarias sobre la actividad pesquera y acuícola. Integrante de la Mesa de Salmón.
Ministerio del Medio

Ambiente (Ley

20417/10) en reemplazo de CONAMA
Diseñar y aplicar políticas, planes y programas en materia ambiental. Proteger y conservar la diversidad biológica y de los recursos naturales renovables e hídricos. Promover el desarrollo sustentable, la integridad de la política ambiental y su regulación normativa.

Subsecretaría de Marina Integrante de la Mesa de Salmón.

Fuente: elaboración propia. 
Tabla 2. Principal normativa relacionada con el desarrollo de la acuicultura de salmones en Chile

\begin{tabular}{|c|c|c|}
\hline Normativa & $\begin{array}{l}\text { Año de vigencia y princi- } \\
\text { pales definiciones }\end{array}$ & $\begin{array}{l}\text { Modificatorias: Año y principales } \\
\text { definiciones }\end{array}$ \\
\hline $\begin{array}{l}\text { Ley General de Pesca y } \\
\text { Acuicultura (LGPA) }\end{array}$ & $\begin{array}{l}\text { Decreto Supremo (D. S.) } \\
\text { N. }{ }^{\circ} 430 \text { de } 1991 \text { fija el } \\
\text { texto refundido, coordi- } \\
\text { nado y sistematizado de } \\
\text { la Ley } 18892 \text { de } 1989 . \\
\text { Áreas Aptas para la } \\
\text { Acuicultura (AAA), } \\
\text { a partir de la cual se } \\
\text { otorgan las concesiones } \\
\text { acuícolas. }\end{array}$ & $\begin{array}{l}\text { 2010: Ley } 20434 \text { (principal } \\
\text { modificación a la Ley 18892): } \\
\text { Introduce áreas de manejo o } \\
\text { zonificación sanitaria (barrios y } \\
\text { macrozonas) y establece scores } \\
\text { de riesgos para establecer la } \\
\text { densidad de siembra permitida } \\
\text { (monitoreo ex antes). } \\
\text { 2013: Ley 20657. }\end{array}$ \\
\hline $\begin{array}{l}\text { Reglamento Ambien- } \\
\text { tal de la Acuicultura } \\
\text { (RAMA) }\end{array}$ & $\begin{array}{l}\text { 2001: regulaciones en } \\
\text { materia ambiental. }\end{array}$ & $\begin{array}{l}\text { 2010: nuevas y más exigentes } \\
\text { regulaciones enfocadas en el } \\
\text { manejo ambiental. }\end{array}$ \\
\hline $\begin{array}{l}\text { Reglamento de Medi- } \\
\text { das de Protección, } \\
\text { Control y Erradicación } \\
\text { de Enfermedades de } \\
\text { Alto Riesgo para las } \\
\text { Especies Hidrobiológi- } \\
\text { cas (RESA) }\end{array}$ & $\begin{array}{l}\text { 2001: regulaciones en } \\
\text { materia sanitaria. }\end{array}$ & $\begin{array}{l}\text { 2010: nuevas y más exigentes } \\
\text { regulaciones enfocadas en el } \\
\text { manejo sanitario. }\end{array}$ \\
\hline
\end{tabular}

Fuente: elaboración propia. 
Tabla 3. Principal normativa relacionada con el desarrollo de la acuicultura en Tierra del Fuego

\begin{tabular}{|c|c|c|}
\hline Normativa & $\begin{array}{l}\text { Año de vigencia y principales } \\
\text { definiciones }\end{array}$ & $\begin{array}{l}\text { Modificatorias: Año y } \\
\text { principales definiciones }\end{array}$ \\
\hline $\begin{array}{l}\text { Ley Provincial de } \\
\text { Pesca }\end{array}$ & $\begin{array}{l}\text { Ley } 244 \text { de } 1995 \\
\text { Respecto a la actividad } \\
\text { acuícola, determinación } \\
\text { de condiciones generales } \\
\text { de la actividad (áreas } \\
\text { de cultivo y especies; } \\
\text { permisos; concesiones; } \\
\text { duración, requisitos), así } \\
\text { como especificación de } \\
\text { comportamiento de los centros } \\
\text { de cultivo (obligatoriedad } \\
\text { de denuncia ante presencia } \\
\text { o presunta presencia de } \\
\text { enfermedades a fin de } \\
\text { erradicarlas y prevenir su } \\
\text { propagación). }\end{array}$ & $\begin{array}{l}\text { 2001: Ley } 537 \\
\text { (modificatoria del art. } 32 \text { de } \\
\text { la Ley 244). } \\
\text { 2007: Decreto } \\
\text { Reglamentario } 670 \text { del } \\
\text { art. } 2 \text { Ley 537. Menciona } \\
\text { explícitamente la } \\
\text { salmonicultura en cuanto } \\
\text { a la localización para el } \\
\text { caso de proyectos que } \\
\text { se desarrollen en agua } \\
\text { dulce (anexo I, punto 5.2). } \\
\text { Especifica que los centros } \\
\text { de cultivo deberán contar } \\
\text { con sistemas de seguridad } \\
\text { a fin de evitar escape de las } \\
\text { especies cultivadas (Anexo } \\
\text { I, punto 10.1 y 10.3). }\end{array}$ \\
\hline
\end{tabular}

Ley Provincial de adhesión a la Ley Nacional (LN) de Desarrollo Sustentable del Sector Acuícola (N. ${ }^{\circ}$ 27231/15)
Ley 1168 y Decreto

Reglamentario 2215 de 2017

Responsabilidad de las autoridades provinciales respecto a la determinación de la "capacidad de carga” o “capacidad de soporte” de los ecosistemas acuáticos (art 7, LN 27231).

Implementación de programas de vigilancia epidemiológica, en colaboración de las provincias y otras entidades involucradas (art 8, LN 27231).

Fuente: elaboración propia. 
Tabla 4. Institucionalidad involucrada con el desarrollo acuícola en Tierra del Fuego

Institución Rol

Ministerio de Agricultura,

Principal organismo regulador y fiscalizador.

Ganadería y Pesca (unidad operativa Dirección General de

Pesca y Acuicultura)

Servicio Nacional de Seguridad

y Calidad Agroalimentaria

SENASA
Prevenir y controlar contingencias y monitoreo, así como normativas que por las características propias de las especies explotadas, sean requeridas para el mantenimiento de la actividad sustentable en todas las cuencas acuícolas del territorio nacional (art. 8, Ley Nacional 27231/15).

Fuente: elaboración propia.

\section{REFERENCIAS BIBLIOGRÁFICA}

Arce J., Lizana V. \& Tagle, Z. (2016). Salmonicultura chilena: análisis de la industria, propuesta de política y estabilización. Recuperado de http://repositorio. uchile.cl/handle/2250/139540

Borello, J. (2015). Complejos productivos y territorio en la Argentina Aportes para el estudio de económica del país. CEPAL. Recuperado de https://repositorio. cepal.org/handle/11362/39227

Bräunin Wisturba, M. \& Rivera Mercado, C. (2017). Reconversión de industrias ligadas a los recursos naturales: la promesa incumplida de la industria salmonera en Chile. Revista Venezolana de Gerencia, 22 (77), 121-136.

Brundtland, G. H. (1987). Informe Brundtland. Comisión Mundial para el Medio Ambiente y el Desarrollo de la ONU [en línea].

Cantero, C. y Romano S. A. (2018). La extracción de árido en Tierra del Fuego. Ponencia presentada para las Primeras Jornadas Argentinas de Desarrollo Territorial. Recuperado de http://fahuweb.uncoma.edu.ar/index.php/academica/departamentos/geografia

Chávez Zúñiga, E. \& Milahuichún Mayorga, C. (2011). Los efectos socioeconómicos de la salmonicultura en la región de Magallanes (Tesis). Universidad de Magallanes, Punta Arenas. Recuperado de https://docplayer. 
es/109733513-Los-efectos-socioeconomicos-de-la-salmonicultura-en-la-region-de-magallanes.html

Escobar, A. (2011). Una minga para el posdesarrollo. Signo y pensamiento, 30 (58), 278-284.

Fine, G. A. \& Deegan, J. G. (1996). Three principles of serendip: insight, chance, and discovery in qualitative research. International Journal of Qualitative Studies in Education, 9 (4), 434-447.

Gereffi, G. (2003). The Governance of Global Value Chains: An Analytic Framework. Review of International Political Economy, 12 (1), 78-104.

Gudynas, E. (2009). Diez tesis urgentes sobre el nuevo extractivismo. En J. Schuldt. Extractivismo, política y sociedad (187-225). Quito: CAAP-CLAES.

Gudynas, E. (2014). Conflicto y extractivismo. Conceptos contenidos y dinámicas. Decursos, Revista en Ciencias Sociales, 27-28, 79-115.

Kaplinsky, R. \& Morris, M. (2009). Un manual para investigación de cadenas de valor. Preparado para el IDRC. Recuperado de https://proyectaryproducir.com.ar/wp-content/uploads/2010/04/Kaplinsky-Manual-completo-Rev-4-2010doc.pdf

Katz, J. \& Araya, C. (2015). Reflexiones en torno al largo plazo de la salmonicultura chilena. Universidad de Chile. Recuperado de http://www.cnid.cl/ portfolio-items/reflexiones-en-torno-al-largo-plazo-de-la-salmoniculturachilena/

Katz, J., Lizuka, M. \& Muñoz, S. (2011). Crecimiento en base a los recursos naturales, "tragedias de los comunes" y el futuro de la industria salmonera chilena”. Serie Desarrollo Productivo no. 191. Chile: CEPAL.

Leef, E. (2002). Saber ambiental: sustentabilidad, racionalidad, complejidad, poder. México: Siglo XXI.

Martínez, I. y Soto, E. (2016). Auditorías de Contraloría a Subpesca y Sernapesca en Materia de Acuicultura. Recuperado de http://repositorio.uchile.cl/handle/2250/141675

Ostrom, E., Burger, J., Field, C. B., Norgaard, R. B. \& Policansky, D. (1999). Revisiting the commons: local lessons, global challenges. Science, 284 (5412), 278-282.

Padilla Pérez, R. (2014). Fortalecimiento de las cadenas de valor como instrumento de la política industrial. En Fortalecimiento de las cadenas de valor como instrumento de la política industrial. Metodología y experiencia de la CEPAL en Centroamérica. Recuperado de http://repositorio.cepal.org:80/ handle/11362/36743

Pérez, C. (2010). Dinamismo tecnológico e inclusión social en América Latina: una estrategia de desarrollo productivo basada en los recursos naturales. Revista Cepal, 100, 123-145. 
Sautu, R., Boniolo, P., Dalle, P. \& Elbert, R. (2005). Manual de metodología: construcción del marco teórico, formulación de los objetivos y elección de la metodología. Buenos Aires: CLACSO.

Svampa, M. (2012). Consenso de los commodities, giro ecoterritorial y pensamiento crítico en América Latina. Osal, 13 (32), 15-38.

Vereda, M. \& Jensen, M. (2014). Territorios remotos del turismo: la articulación de espacios turísticos asociados en el Atlántico Sur Meridional. Universidad Nacional de Tierra del Fuego.

Zanlungo, M., Pablo, J., Katz, J. \& Araya, G. (2015). Servicios intensivos en conocimiento en la industria salmonera chilena. Inter-American Development Bank. Recuperado de https://publications.iadb.org/publications/spanish/ document/Servicios-intensivos-en-conocimiento-en-la-industria-salmonera-chilena.pdf

(C) 2020 por los autores; licencia no exclusiva otorgada a la revista Estudios económicos. Este artículo es de acceso abierto y distribuido bajo los términos y condiciones de una licencia Atribución-No Comercial 4.0 Internacional (CC BY-NC 4.0) de Creative Commons. Para ver una copia de esta licencia, visite http://creativecommons.org/licenses/by-nc/4.0 